\title{
A Fair Resource Sharing Mechanism between Mobile Virtual Network Operators
}

\section{Wei LIU $^{\dagger \text { a) }}$, Rui HU ${ }^{\dagger}$, Student Members, Ryoichi SHINKUMA ${ }^{\dagger}$, Senior Member, and Tatsuro TAKAHASHI ${ }^{\dagger}$, Fellow}

\begin{abstract}
SUMMARY Mobile virtual network operators (MVNOs) are mobile operators without their own infrastructure or government issued spectrum licenses. They purchase spectrum resources from primary mobile network operators (MNOs) to provide communication services under their own brands. MVNOs are expected to play an important role in mobile network markets, as this will increase the competition in retail markets and help to meet the demand of niche markets. However, with the rapidly increasing demand of mobile data traffic, efficient utilization of the limited spectrum resources owned by MVNOs has become an important issue. We propose here a resource sharing mechanism between MVNOs against the background of network functions virtualization (NFV). The proposed mechanism enables MVNOs to improve their quality of service (QoS) by sharing spectrum resources with each other. A nash bargaining solution based decision strategy is also devised to ensure the fairness of resource sharing. Extensive numerical evaluation results validate the effectiveness of the proposed models and mechanisms.

key words: mobile virtual network operator, fair resource sharing, network functions virtualization
\end{abstract}

\section{Introduction}

Mobile virtual network operators (MVNOs) are defined as mobile operators without their own infrastructure or government issued spectrum licenses. MVNOs purchase spectrum resources from primary mobile network operators (MNOs), and provide services to end customers under their own brands. Governments expect authorized MVNOs to increase competition in the retail market as well as to meet the demands of niche markets. Nowadays, MVNOs have spread throughout the world and have become an important part of mobile network markets. For example, Virgin Mobile in the United States is providing services to more than 4 million users [1].

Advanced communication technologies such as the third generation of mobile telecommunications technology (3G) and Long Term Evolution (LTE) have resulted in skyrocketing numbers of subscribers to mobile data services recently. Mobile data traffic is forecast to increase 13 times by 2017 , with the volume climbing to 13.2 exabytes per month ${ }^{*}$ and the number of users approaching approximately 5.2 billion [2]. Therefore, determining how to maintain (or improve) the quality of service (QoS) for end users with relatively limited spectrum resources has become an impor-

Manuscript received March 25, 2015.

Manuscript revised June 26, 2015.

${ }^{\dagger}$ The authors are with Graduate School of Informatics, Kyoto University, Kyoto-shi, 606-8501 Japan.

a)E-mail: liu@cube.kuee.kyoto-u.ac.jp

DOI: 10.1587/transcom.E98.B.2141

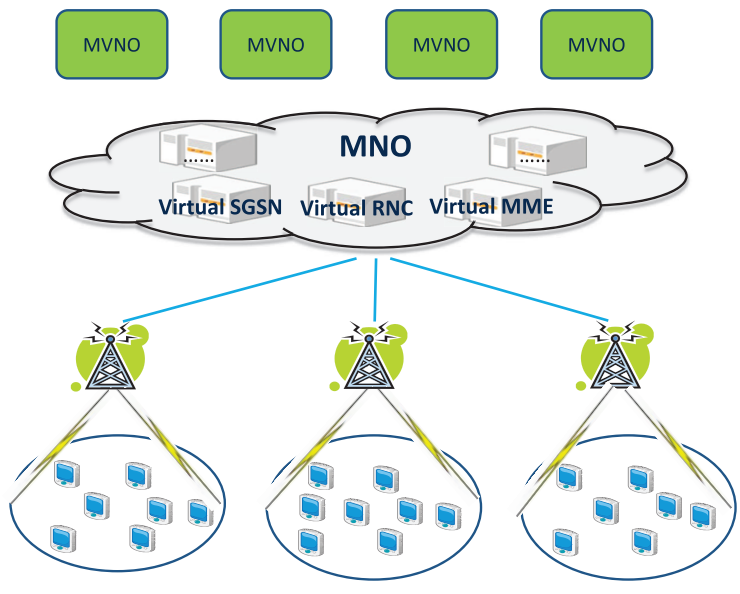

Fig. 1 Application scenarios.

tant issue for both MNOs and MVNOs. Frisanco et al. [3] demonstrated that spectrum resource sharing could be an effective strategy in dealing with this issue. Previous efforts focused mainly on spectrum resource sharing between MNOs. Unfortunately, since different MNOs own different physical network infrastructures that are difficult to integrate effectively, resource sharing between MNOs has not been widely accepted by the market. Compared with existing proposals, we argue resource sharing between MVNOs belonging to the same MNO would be more feasible from technical, economic, and security view points, since these MVNOs are already running on the same suite of network infrastructure products**.

Recent research progress in network functions virtualization (NFV) [4] makes the idea of spectrum resource sharing between MVNOs more realistic than ever before. NFV consolidates mobile network nodes such as service GPRS support node (SGSN), radio network controller (RNC), and mobility management entity (MME) onto industry standard high volume servers, and controls these nodes with management software. Therefore, spectrum resources can be optimally allocated between MVNOs in near real time, according to the actual traffic and service demands. Figure 1 illustrates the application scenarios of MVNOs under NFV.

\footnotetext{
"Mainly from "heavy applications" such as video games and streaming media.

** The MNO itself can also be treated as a special MVNO running on its own infrastructure.
} 
We propose in this paper a fair mechanism based on previous observations, to improve the QoS of MVNOs by sharing their spectrum resources. The three main contributions of this paper are: (1) Models of resource sharing between MVNOs are presented; (2) A fair resource sharing mechanism is introduced to incentivize MVNOs to participate in resource sharing; and (3) Numerical evaluation results have validated the effectiveness of the proposed models and mechanism.

Following the literature review in Sect. 2, Sect. 3 explains a resource sharing model between MVNOs and presents numerical evaluations of the model. Analyses of the fair resource sharing mechanism and its corresponding numerical evaluations are explained in Sect. 4. Conclusions are drawn and future work is discussed in the last section.

\section{Related Work}

There is a great deal of related work on this subject because of its importance. However, most research has focused on pricing mechanisms of MVNOs rather than their QoS. Cadre et al. [5] proposed a pricing model in which an MNO shared its network infrastructures with an MVNO. A game was generated in which the MVNO invested in content/advertising to compensate for the QoS degradation of MNO. Pattanavichai et al. [6] analyzed the optimal decision strategies to maximize the expected profit values for both of the MNO and the MVNO. Distinguished from previous two papers that worked on games between an MNO and MVNO pair, Zhao et al. [7] proposed a model for pricing competition between two MVNOs. The bertrand game theory was adopted to address issues of dynamic spectrum access, and backward induction was used to achieve the unique equilibrium of the game.

So far, there has been very little work on the QoS of MVNOs. Hong et al. [8] presented a femtocell-based model in which both MNO and MVNO shared spectrum resources to enable mobile users to roam between them. Their work investigated the interaction between the transmit power strategy of the MVNO and the absorbing behavior for the subscribers of its MNO. However, their strategy degraded the QoS of the MVNO to benefit the MNO. Since MVNOs and the MNO may also be competitors in the retail market, their strategy is less attractive to MVNOs. Bedhiaf et al. [9] confirmed that MVNOs achieved a better gain in deployment time by virtualizing network components rather than physically installing them. To the best of our knowledge, no existing work discusses spectrum resource sharing between MVNOs.

\section{Resource Sharing between MVNOs}

\subsection{System Model}

We focus on spectrum sharing between two MVNOs in this paper, and we leave the one involving more MVNOs as future work. Without loss of generality, $M V N O_{i}$ and $M V N O_{j}$

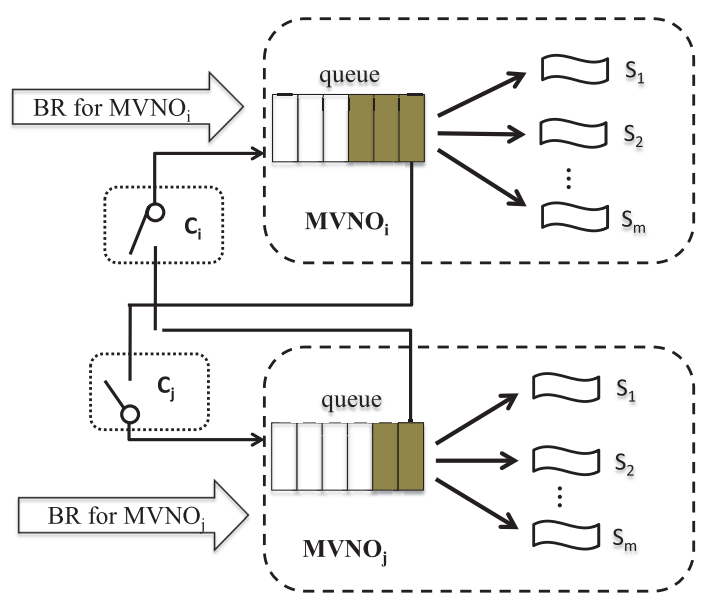

Fig. 2 System model.

are used to represent the two MVNOs in the following descriptions. As shown in Fig. 2, each MVNO splits its spectrum resources into units of subcarriers represented by $S_{i}$, e.g., both MVNOs own $m$ units of subcarriers in Fig. 2. In the conventional strategy, when an end-user $u_{i}$ of $M V N O_{i}$ generates a bandwidth request (BR) for a mobile application such as voice over IP (VoIP) or video streaming, $M V N O_{i}$ allocates one unit of the subcarrier to process his BR. ${ }^{\dagger} \mathrm{An} B R$ should be blocked for admission in a first-in-first-out (FIFO) queue, if all subcarriers of $M V N O_{i}$ are already occupied. Admission delay (AD) is defined to measure the length of time that an BR is blocked in the queue.

With the resource sharing strategy, two MVNOs can share spectrum resources with each other. Without loss of generality, assume that all subcarriers of $M V N O_{i}$ have been occupied, and $B R_{i}$ arrives to request its bandwidth. Instead of blocking $B R_{i}$ in the queue, $M V N O_{i}$ sends a cooperation request (CR) to $M V N O_{j}$. If $M V N O_{j}$ still has idle subcarriers and prefers to cooperate with $M V N O_{i}, B R_{i}$ is unblocked from the queue and is redirected to $M V N O_{j}$ to utilize its idle subcarrier. Otherwise, $B R_{i}$ continues to be blocked in the queue for the next available subcarrier of $M V N O_{i} . C_{k}$ is used to indicate whether $M V N O_{k}$ prefers to cooperate with the other MVNO:

$$
C_{k}= \begin{cases}C & \text { Cooperation, } \\ N & \text { Non-cooperation, }\end{cases}
$$

e.g., $C_{i}$ and $C_{j}$ shown in Fig. 2 indicate the attitudes of $M V N O_{i}$ and $M V N O_{j}$.

Obviously, the average admission delay (AAD) of BRs is one of the most important metrics that reveal the QoS provided by MVNOs, since a long delay degrades users' experiences significantly. ${ }^{\dagger \dagger}$ Therefore, it would be meaningful to

${ }^{\dagger}$ This is a simplified fixed bandwidth allocation (FBA) model as adopted by Falowo et al. [10]. We assume that the bandwidth of the subcarrier satisfies the minimum QoS constraint of the application.

${ }^{\dagger}$ Both AAD and bandwdith affect the QoSs provided by 
verify that whether the resource sharing strategy is capable of decreasing AAD to improve MVNOs' QoSs.

\subsection{Numerical Evaluation Model}

There are three major factors affect the AADs of MVNOs in the proposed system model. They are explained here and were also used in the numerical evaluations described in Sect. 3.3.

\subsubsection{Arrival Pattern of Data Traffic}

The arrival pattern of data traffic defines how often mobile users demand spectrum resources to deal with generated BRs. One of the most common patterns is a Poisson process that generates BRs with intervals conforming to exponential distributions. However, Paxson et al. [11] have proved that it fails to match real scenarios.

Leland et al. [12] have illustrated that arrivals of data traffic are statistically "self-similar", which is in contrast to the Poisson process. ${ }^{\dagger}$ Therefore, we used fractional brownian motion (FBM) [13], one of the most popular analytical models to simulate a self-similar data process in our evaluations. The number of BRs generated in the time interval [0, t), $A(t)$, is given by.

$$
A(t)=m t+\sqrt{\alpha m} Z_{H}(t), \quad t>0,
$$

where $m$ indicates the average number of BRs generated in one unit of time, and $\alpha$ represents the variance coefficient of $A(t)$. Finally, $Z_{H}(t)$ is a standard FBM process, where the hurst parameter $H$ determines the degree of self-similarity. Details of the FBM model have not been explained in this paper because they are beyond its scope. However, a detailed description is available in [13].

\subsubsection{Size of Data Traffic}

The size of data traffic affects the time taken to process an BR, e.g., data with a larger size require a longer transmission time. A log-normal distribution was used for the size of data traffic in our evaluations as suggested by Antoniou et al. [14]. Its probability density function $p(x)$ is given by

$$
p(x)=\frac{1}{x \sigma \sqrt{2 \pi}} e^{-\frac{(\ln x-\mu)^{2}}{2 \sigma^{2}}}, x>0,
$$

where $\mu$ and $\sigma$ are the mean and the standard deviation (SD) of the size of the generated data traffic.

\subsubsection{State of Wireless Channel}

A lognormal fading model was used to simulate the slow MVNOs. Since we assume each user is allocated with a fixed amount of bandwidth (a unit of subcarrier), we focus on reducing AAD to improve the QoSs. Other factors like the transmitting power and antenna gains are beyond the scope of this paper.

†"Self-similar" means that an object is exactly or approximately similar to a part of itself, e.g., parts of coastlines often exhibit the same statistical properties at many scales. fading of wireless channels in our evaluations. Without loss of generality, $P_{t}$ and $P_{r}$ are used to represent the transmit power of base stations and the receive power of mobile endusers in the following descriptions. In the lognormal fading model, the transmit-to-receive power ratio $\psi=P_{t} / P_{r}$ is a random variable that follows a lognormal distribution [15] and is given by

$$
p(\psi)=\frac{\xi}{\sqrt{2 \pi} \sigma_{\psi_{d B}} \psi} \exp \left[-\frac{\left(10 \lg \psi-\mu_{\psi_{d B}}\right)^{2}}{2 \sigma_{\psi_{d B}}^{2}}\right],
$$

where $\xi=\frac{10}{\ln 10}$, and $\mu_{\psi_{d B}}$ and $\sigma_{\psi_{d B}}$ are the mean and the SD of $\psi$ in $\mathrm{dB}$.

Finally, the capacity of additive white Gaussian noise (AWGN) channels is used for the throughput of data transmission,

$$
C_{A W G N}=B \log _{2}\left(1+\frac{P_{r}}{N_{0} \times B}\right),
$$

where $B$ is the bandwidth and $N_{0} / 2$ is the power spectral density of the noise.

\subsection{Numerical Results for Resource Sharing}

Matlab 7.0 was used to evaluate the effectiveness of the proposed resource sharing mechanism. Two MVNOs $\left(M V N O_{i}\right.$ and $M V N O_{j}$ ) were located on the same base station owned by their MNO. Both MVNOs divided their spectrum resources into 10 units of subcarriers and each subcarrier occupies a bandwidth of $200 \mathrm{kHz}$. The FBM model was used to simulate traffic arrival patterns in the evaluation. The average number of generated BRs for $M V N O_{i}\left(\lambda_{i}\right)$ varied from 1 to 9 in one second, while that of $M V N O_{j}\left(\lambda_{j}\right)$ was fixed at 8.5. The data size of each BR followed a lognormal distribution, with the average size $\mu=1$ Mbits and the $\operatorname{SD} \sigma=0.2$. For the wireless transmission channels, the transmit power of the base station was set to be $20 \mathrm{~W}$, while the transmit-toreceive power ratio $\psi$ followed a lognormal distribution with the average $\mu_{\psi_{d B}}=10 \mathrm{~dB}$ and the SD $\sigma_{\psi_{d B}}=6$. The noise power spectral density $N_{0}$ was $10^{-9} \mathrm{~dB}$. All parameters used in the numerical evaluation are summarized in Table 1. Finally, it should be noted that these parameter settings are somewhat arbitrary, but plausible, e.g., we assume that a bandwidth of $200 \mathrm{kHz}$ satisfies the minimum QoS constraint of the focused mobile application such as VoIP and streaming media. Other settings do not affect any conclusion made in the rest of this paper. However, since queueing models are used in the system as shown in Fig. 2, the values of $\lambda_{i}$ and $\lambda_{j}$ should not be beyond the processing capacity of their corresponding MVNOs. ${ }^{\dagger \dagger}$

As indicated in Eq. (1), both MVNOs have two different strategies when they receive a cooperation request (CR)

\footnotetext{
${ }^{\dagger}$ The processing capacity of one MVNO is mainly determined by factors like its number of subcarriers, the bandwidth of its subcarriers, the average size of generated data traffic, and the throughput of its transmission channels.
} 
Table 1 Simulation parameters.

\begin{tabular}{ll}
\hline Simulation period & $100000 \mathrm{~s}$ \\
Number of subcarriers & 10 \\
Bandwidth of each subcarrier & $200 \mathrm{kHz}$ \\
Number of generated BRs $\left(M V N O_{i}\right), \lambda_{i}$ & 1 to 9 per second \\
Number of generated BRs $\left(M V N O_{j}\right), \lambda_{j}$ & 8.5 per second \\
Hurst parameter H & 0.8 \\
Average size of data traffic $\mu$ & $1 \mathrm{Mbit}$ \\
SD of data traffic $\sigma$ & 0.2 \\
Average transmit-to-receive ratio $\mu_{\psi_{d B}}$ & $10 \mathrm{~dB}$ \\
SD of transmit-to-receive ratio $\mu_{\psi_{d B}}$ & 6 \\
Noise power spectral density & $10^{-9} \mathrm{~dB}$ \\
Transmission power of base station & $20 \mathrm{~W}$ \\
\hline
\end{tabular}

Table 2 Strategies of MVNOs.

\begin{tabular}{|c|c|c|}
\hline $\begin{array}{ll}M V N O_{i} & M V N O_{j} \\
\end{array}$ & Cooperation & Non-cooperation \\
\hline Cooperation & $(\mathrm{C}, \mathrm{C})$ & $(\mathrm{C}, \mathrm{N})$ \\
\hline Non-cooperation & $(\mathrm{N}, \mathrm{C})$ & $(\mathrm{N}, \mathrm{N})$ \\
\hline
\end{tabular}

from the other. Consequently, there are four different combinations of their strategies as shown in Table 2. Obviously, three of them represent different cooperation scenarios between the two MVNOs, i.e. $(\mathrm{C}, \mathrm{N}),(\mathrm{N}, \mathrm{C})$, and $(\mathrm{C}, \mathrm{C})$, while $(\mathrm{N}, \mathrm{N})$ indicates there is no cooperation between them.

Figure 3(a) indicates AADs of both MVNOs in the scenario $(\mathrm{C}, \mathrm{N})$ against their AADs in the scenario $(\mathrm{N}$, $\mathrm{N})$. Compared to the scenario without any cooperation, the AAD of $M V N O_{j}$ in $(\mathrm{C}, \mathrm{N})$ decreased while that of $M V N O_{i}$ increased. The AAD of $M V N O_{j}$ decreased because $M V N O_{i}$ accepted CRs from $M V N O_{j}$ to process its blocked BRs. On the other hand, since $M V N O_{j}$ rejected any CR from $M V N O_{i}, M V N O_{i}$ not only dealt with all of its own BRs but also had to process extra BRs redirected by $M V N O_{j}$. Therefore, the AAD of $M V N O_{i}$ increased. Since the values of AAD of $M V N O_{i}$ are suppressed by larger values in the figure when $\lambda_{i}$ is small, these values are shown seperately in Fig. 3(b) to make their comparisons clear. Similar results are shown in Figs. 4(a) and (b) that indicates AADs of both MVNOs in the scenario $(\mathrm{N}, \mathrm{C})$ against their AADs in the scenario $(\mathrm{N}, \mathrm{N})$, except the roles of two MVNOs were reversed.

Figure 5(a) plots AADs of two MVNOs in the scenario $(\mathrm{C}, \mathrm{C})$ against their AADs in the scenario $(\mathrm{N}, \mathrm{N})$. Again, the values of AAD of $M V N O_{i}$, when $\lambda_{i}$ is small, are shown seperately in Fig. 5(b) to make their comparisons clear. The results are two-fold:

\section{(1) When $\lambda_{i}<7$}

The AAD of $M V N O_{i}$ increased, while that of $M V N O_{j}$ decreased. Since the load of $M V N O_{j}$ is much higher than that of $M V N O_{i}$, the direction of most CRs is from $M V N O_{j}$ to $M V N O_{i}$. Therefore, in most time of the evaluation period, $M V N O_{i}$ acted as a resource provider, while $M V N O_{j}$ acted as a resource requester. Obviously, $M V N O_{j}$ benefited from resource sharing, while $M V N O_{i}$ only suffered its side effects in this case.

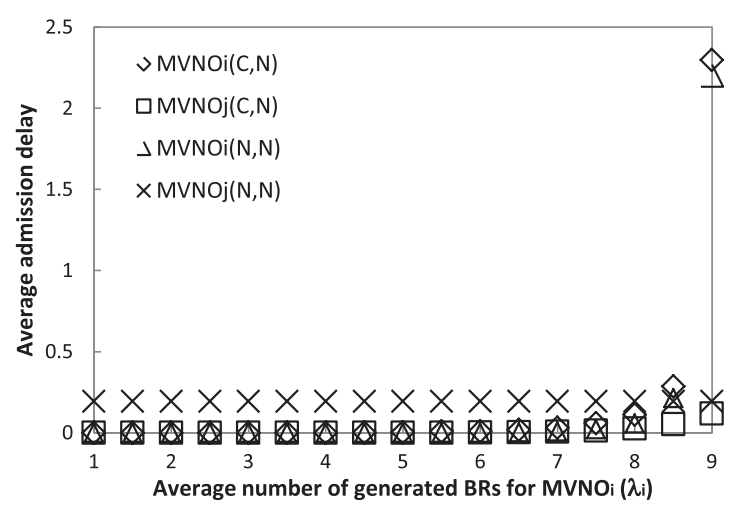

(a)

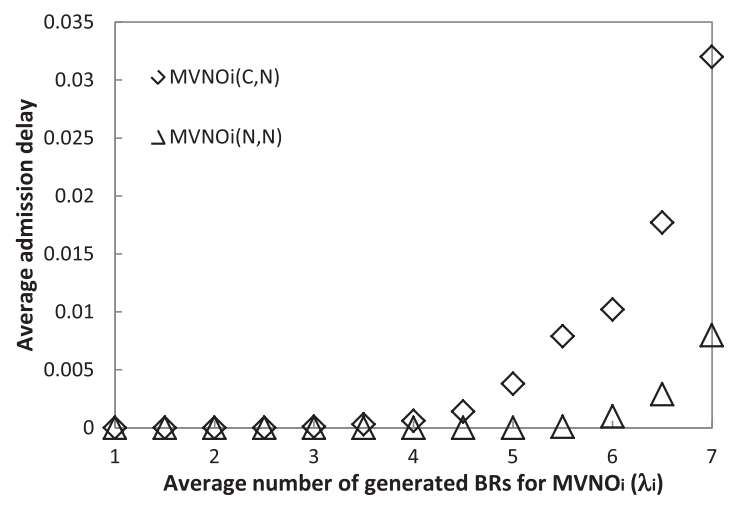

(b)

Fig. 3 Average admission delay, (C, N) against (N, N).

(2) When $\lambda_{i} \geq 7$

The AADs of both MVNOs decreased. As $\lambda_{i}$ increases, loads of two MVNOs and the number of CRs generated by each of them were close. Both MVNOs have a similar possibility to be a resource provider or a resource requester in the evaluation period. Therefore, two MVNOs fairly benefited from the cooperation strategy and improved their QoS.

\section{Fair Resource Sharing between MVNOs}

As indicated by the numerical results in the previous section, the effectiveness of resource sharing depends on different scenarios; it may either improve the QoS of one MVNO while sacrificing that of the other MVNO or improve the QoS of both MVNOs efficiently. Because MVNOs are competitors in the retail market, no participating MVNO is motivated to share its spectrum resources with competitors without any benefit. As a result, this section proposes an Nash bargaining solution (NBS) based decision strategy to ensure fair resource sharing between MVNOs, which is essential to apply the proposed resource sharing mechanism in the real market. 


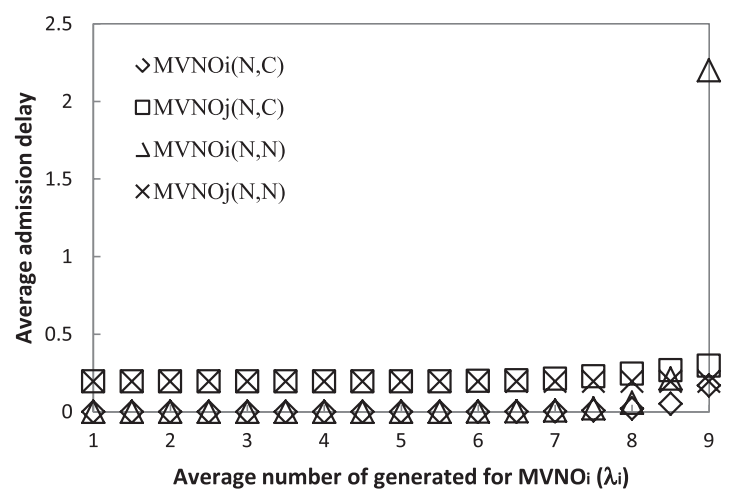

(a)

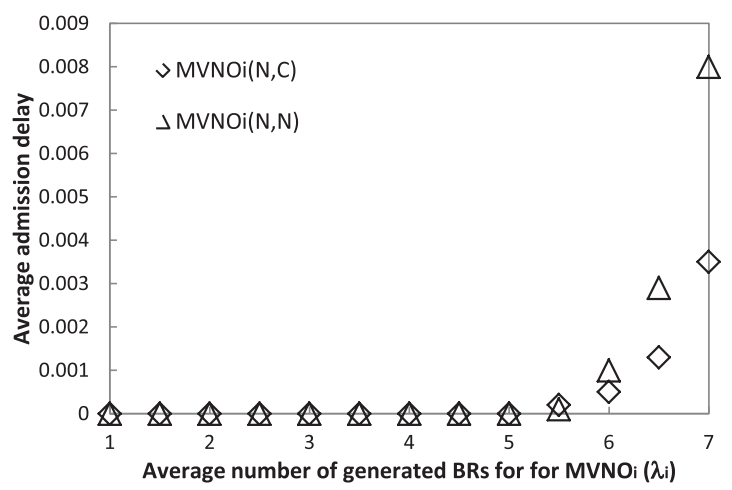

(b)

Fig. 4 Average admission delay, (N, C) against (N, N).

\subsection{Analysis of Fairness in Resource Sharing}

The numerical evaluation results shown in Fig. 5 show that even when both MVNOs adopted the cooperation strategy, the unfairness problem still arose when there was a large gap between $\lambda_{i}$ and $\lambda_{j}$. Actually, it is similar to the concept of "give-and-take" in our daily lives. Assume that there are two persons, Bob and Alice. Sometimes, Bob has a trouble and Alice helps him. Some other times, Bob also gives his hand to Alice and helps her to overcome her troubles. As a result, there are two kinds of outcomes:

(1) Bob always gives his hand to Alice, but he seldom needs Alice's help. Obviously, Alice benefits from the cooperation since she solves her troubles by Bob's help. However, since Bob has to take care of extra troubles from Alice, he does not have any benefit. Similar results can be given if they change their roles.

(2) Bob always gives his hand to Alice, and he also requests for Alice's help with a similar frequency. Then, both of them can benefit from the cooperation because it is fair (not one-sided, but two-sided).

Suppose Bob and Alice are competitors like two MVNOs in our paper and they only consider their own ben-

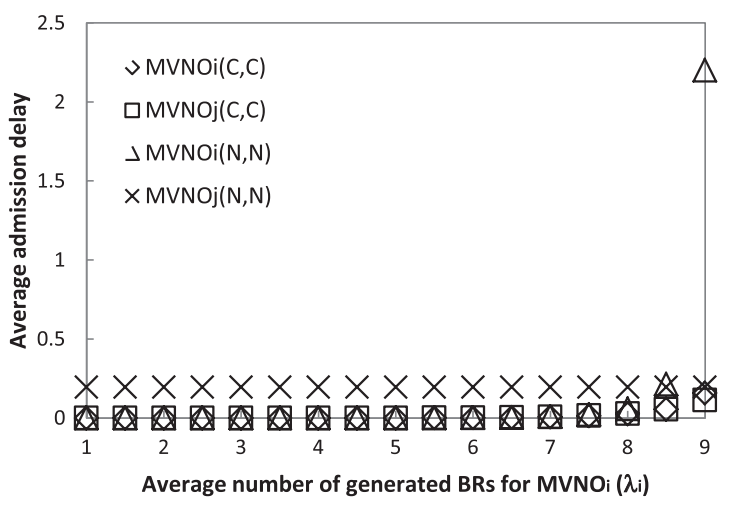

(a)

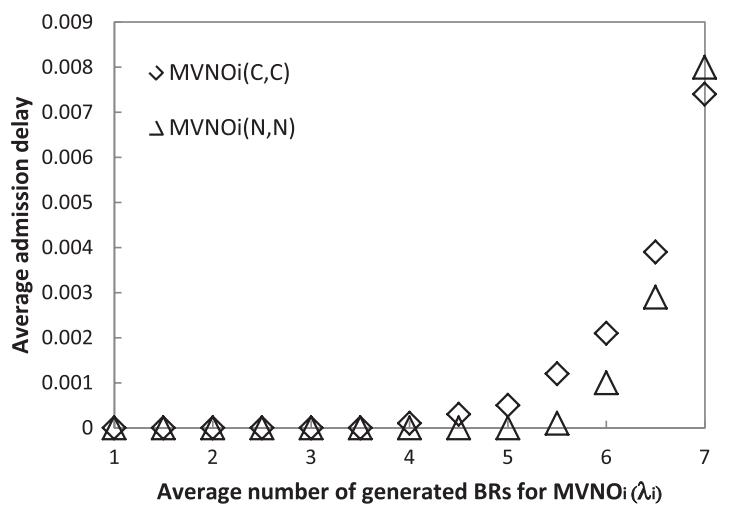

(b)

Fig. 5 Average admission delay, (C, C) against (N, N).

efits to decide whether to cooperate with each other or not. Definitely, a mechanism that ensures benefits of both participants is essential to motivate Bob and Alice (two MVNOs) to join the cooperation. The following subsections formalize this problem into a game and devise an NBS-based decision strategy to achieve fair resource sharing.

\subsection{Formalization of Fair Resource Sharing}

As described in Sect. 3.1, MVNOs participate in resource sharing to reduce their AADs. Therefore, the utility of each MVNO can be measured by the difference between its AAD with resource sharing and its AAD without any resource sharing. Without loss of generality, $u_{k t}$ is defined to represent this difference,

$$
u_{k t}=A A D_{k 4}-A A D_{k t},
$$

where $k$ represents the ID of MVNO, $t$ represents different scenarios as indicated in Table 3 , and $A A D_{k t}$ represents the corresponding AAD of $M V N O_{k}$ under scenario $t$. For example, $u_{i 1}=A A D_{i 4}-A A D_{i 1}$ represents the utility of participating in resource sharing for $M V N O_{i}$ under the scenario $(\mathrm{C}, \mathrm{N})$, which equals to its reduction of AAD compared 
Table 3 Utility of MVNO in resource sharing.

\begin{tabular}{|c|c|c|c|c|}
\hline & $(\mathrm{C}, \mathrm{N})$ & $(\mathrm{N}, \mathrm{C})$ & $(\mathrm{C}, \mathrm{C})$ & $(\mathrm{N}, \mathrm{N})$ \\
\hline$M V N O_{i}$ & $u_{i 1}$ & $u_{i 2}$ & $u_{i 3}$ & $u_{i 4}$ \\
\hline$M V N O_{j}$ & $u_{j 1}$ & $u_{j 2}$ & $u_{j 3}$ & $u_{j 4}$ \\
\hline
\end{tabular}

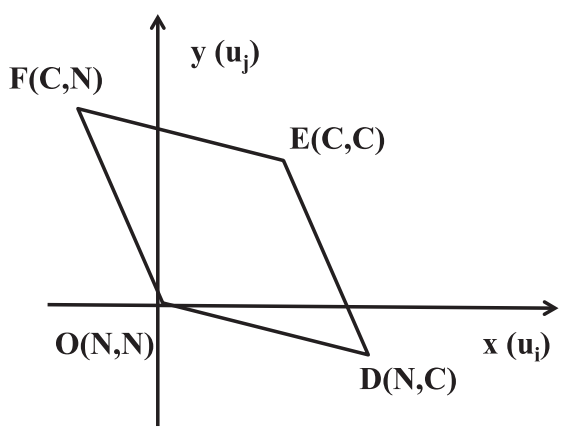

Fig. 6 Feasible strategies and resulting utilities of two MVNOs.

with the scenario of no resource sharing $(\mathrm{N}, \mathrm{N})$. Obviously, $u_{i 4}=u_{j 4}=0$, which means the utilities of both MVNOs are always zero if they never participate in resource sharing.

As shown in Eq. (1), each MVNO has two different choices, i.e., cooperation and non-cooperation. These two choices are named pure strategies in the game theory, since they represent a deterministic choice of the strategy, i.e., accept (the cooperation strategy) or reject (the noncooperation strategy) CRs from the other MVNO constantly. Other than pure strategies, each MVNO can also adopts a mixed strategy that is an assignment of a probability to every pure strategy, i.e. accepts CRs from the other with a probability of $P_{\text {coop }}$, while rejecting them with a probability of $1-P_{\text {coop }}$. Obviously, pure strategies are special cases of mixed strategies when $P_{\text {coop }}=0$ or 1 .

Figure 6 gives a geometric interpretation of two MVNOs' feasible strategies and their resulting utilities. Four vertices, $F, D, E$ and $O$, represent utilities of both MVNOs under four combinations of pure strategies shown in Table 2. For example, the vertex $F$ represents the utilities of both MVNOs in the scenario $(\mathrm{C}, \mathrm{N})$, i.e. $u_{i 1}$ and $u_{j 1}$ defined in Table 3. As shown by the results of Fig. 3, $u_{i 1}$ is negative, while $u_{j 1}$ is positive. Therefore, $F$ locates in the second quadrant of the axes. From the general view of mixed strategies, since both MVNOs accept or reject CRs according to their values of $P_{\text {coop }}$, four scenarios shown in Table 2 emerge randomly. Consequently, feasible utilities of both MVNOs are represented by a plane defined by the four vertices. Without loss of generality, assume that the probabilities of each emerging scenario are $p_{1}, p_{2}, p_{3}, p_{4}$ as indicated in Table 4. Consequently, the expected utility of each MVNO is:

$$
\begin{aligned}
u_{k} & =u_{k 1} p_{1}+u_{k 2} p_{2}+u_{k 3} p_{3}+u_{k 4} p_{4} \\
& =u_{k 1} p_{1}+u_{k 2} p_{2}+u_{k 3} p_{3},
\end{aligned}
$$

where $k$ represents the ID of MVNOs.

The NBS is aimed at maximizing the product of utilities of both MVNOs to ensure the fairness of resource shar-
Table 4 Probability of different scenarios.

\begin{tabular}{|l|c|c|}
\hline$M V N O_{i}$ & Non-cooperation & Cooperation \\
\hline Cooperation & $p_{1}$ & $p_{3}$ \\
\hline Non-cooperation & $p_{4}$ & $p_{2}$ \\
\hline
\end{tabular}

ing,

$$
\begin{aligned}
\max _{p_{1}, p_{2}, p_{3}, p_{4}} & u_{i} \times u_{j} \\
\text { s.t. } & u_{i}, u_{j} \geq 0 \\
& p_{1}+p_{2}+p_{3}+p_{4}=1 \\
& p_{1}, p_{2}, p_{3}, p_{4} \geq 0 .
\end{aligned}
$$

where the constraint $u_{i}, u_{j} \geq 0$ ensures that neither MVNOs degrades its QoS by participating in resource sharing. This optimization problem can also be interpreted by the help of Fig. 6. If part of the plane in Fig. 6 locates in the first quadrant like the point $\mathrm{E}$, there exist feasible cooperation strategies that enable both MVNOs to obtain positive utilities. Therefore, Eq. (8) aims finding the optimal strategies of two MVNOs to maximize their production of utilities. However, if there is no point of this plane locates in the first quadrant, it means at least one of the MVNOs obtains negative utility in the cooperation no matter with the strategies of two MVNOs. Since both MVNOs do not want to obtain negative utilities in the cooperation, they simply choose the pure non-cooperation strategy to maintain a utility of zero (represented by the point $O$ in the figure).

Subscripts $x$ and $y$ are used to denote the horizontal and vertical coordinates of a point, i.e., $D=\left(D_{x}, D_{y}\right), E=\left(E_{x}\right.$, $\left.E_{y}\right)$ and $F=\left(F_{x}, F_{y}\right)$. Define $\tan D=\frac{D_{y}}{D_{x}}$ and define $\tan E$, $\tan F$ similarly. Define $q_{1}=p_{1}+p_{3}$ and $q_{2}=p_{2}+p_{3}$. Zhang et al. [16] have derived their closed-form expressions:

$$
\begin{aligned}
& q_{1}= \begin{cases}0 & \tan D>|\tan F| \\
-\frac{1}{2}\left(\frac{D_{x}}{F_{x}}+\frac{D_{y}}{F_{y}}\right) & |\tan E|>|\tan F|>|\tan D| \\
1 & \text { Otherwise }\end{cases} \\
& q_{2}= \begin{cases}0 & \cot F>|\cot D| \\
-\frac{1}{2}\left(\frac{F_{x}}{D_{x}}+\frac{F_{y}}{D_{y}}\right) & |\cot E|>|\cot D|>|\cot F| \\
1 & \text { Otherwise }\end{cases}
\end{aligned}
$$

The detailed proofs of these two equations have not been explained in this paper because they are beyond its scope and are available in [16].

The values of $p_{1}, p_{2}, p_{3}$, and $p_{4}$ are calculated based on the values of $q_{1}$ and $q_{2}$. There are two different cases:

(1) Both $q_{1}$ and $q_{2}$ are zero:

The optimal solution converges to the point of $\left(p_{1}=0\right.$, $\left.p_{2}=0, p_{3}=0, p_{4}=1\right)$, and the optimal value is zero. This means at least one of the MVNOs obtains negative utility in the cooperation constantly. They choose the pure noncooperation strategy to maintain a utility of zero. 
(2) Either $q_{1}$ or $q_{2}$ is positive:

This means it is possible for both MVNOs to obtain positive utilities from the cooperation. The remaining issue is finding strategies for both MVNOs to achieve the optimal point of Eq. (8).

Theorem 1. If either $q_{1}$ or $q_{2}$ is positive, $p_{4}=0$ in at least one of the optimal points of Eq. (8).

Proof. Assume that there is an optimal point of Eq. (8) with a positive $p_{4}, \mathrm{~S}=\left[p_{1}, p_{2}, p_{3}, p_{4}\right]$, that maximizes $u_{i} \times u_{j}$. Define a new point $S^{\prime}$ with $p_{1}^{\prime}=p_{1}\left(1+\frac{p_{4}}{p_{1}+p_{2}+p_{3}}\right), p_{2}^{\prime}=$ $p_{2}\left(1+\frac{p_{4}}{p_{1}+p_{2}+p_{3}}\right), p_{3}^{\prime}=p_{3}\left(1+\frac{p_{4}}{p_{1}+p_{2}+p_{3}}\right)$, and $p_{4}^{\prime}=0$. Then, the utility value of $M V N O_{i}$ on the point $S^{\prime}$ is

$$
\begin{aligned}
u_{i}^{\prime} & =u_{i 1} p_{1}^{\prime}+u_{i 2} p_{2}^{\prime}+u_{i 3} p_{3}^{\prime} \\
& =\left(1+\frac{p_{4}}{p_{1}+p_{2}+p_{3}}\right)\left(u_{i 1} p_{1}+u_{i 2} p_{2}+u_{i 3} p_{3}\right) \\
& =\left(1+\frac{p_{4}}{p_{1}+p_{2}+p_{3}}\right) u_{i} \\
& \geq u_{i} .
\end{aligned}
$$

The last step comes from the constraint of Eq. (8), i.e., both $u_{i}$ and $u_{j}$ are non-negative. Similarly, it is easy to prove that $u_{j}^{\prime} \geq u_{j}$. Consequently, $u_{i}^{\prime} \times u_{j}^{\prime} \geq u_{i} \times u_{j}$.

$$
\text { (1) } u_{i}^{\prime} \times u_{j}^{\prime}=u_{i} \times u_{j}
$$

Point $S^{\prime}$ achieves the same objective value as $S$. Therefore, $S^{\prime}$ is one of the optimal points of Eq. (8). The proof is complete.

$$
\text { (2) } u_{i}^{\prime} \times u_{j}^{\prime}>u_{i} \times u_{j}
$$

Point $S^{\prime}$ achieves a better objective value than $S$, which contradicts the hypothesis. The proof is complete.

On the basis of Theorem 1, the following four equations are possible:

$$
\left\{\begin{array}{l}
q_{1}=p_{1}+p_{3} \\
q_{2}=p_{2}+p_{3} \\
p_{4}=0 \\
p_{1}+p_{2}+p_{3}+p_{4}=1
\end{array}\right.
$$

By solving these equations, we find that the optimal point of Eq. (8) is $\left[p_{1}=1-q_{2}, p_{2}=1-q_{1}, p_{3}=q_{1}+q_{2}-1, p_{4}=0\right]$. Finally, the result of Eq. (8) is pareto optimal, i.e., neither MVNO can improve its utility without reducing the utility of the other, since it achieves the maximum production value of two MVNOs' utilities.

\subsection{NBS-Based Decision Strategy}

The findings from previous discussion suggest that both MVNOs should accept CRs from the other MVNO with probabilities matched to the optimal solution of Eq. (8) to achieve fair resource sharing. Suppose that the number of CRs received by $M V N O_{i}$ from $M V N O_{j}$ in different scenarios is defined in Table 5.

The number of expected CRs that are received by
Table 5 Number of CRs received by $M V N O_{i}$

\begin{tabular}{|c|c|}
\hline & Number of received CRs \\
\hline$(\mathrm{C}, \mathrm{N})$ & $n_{i 1}$ \\
\hline$(\mathrm{N}, \mathrm{C})$ & $n_{i 2}$ \\
\hline$(\mathrm{C}, \mathrm{C})$ & $n_{i 3}$ \\
\hline$(\mathrm{N}, \mathrm{N})$ & $n_{i 4}$ \\
\hline
\end{tabular}

$M V N O_{i}$ in the fair resource sharing status is given by,

$$
E_{i r}=p_{1} \times n_{i 1}+p_{2} \times n_{i 2}+p_{3} \times n_{i 3}+p_{4} \times n_{i 4} .
$$

$M V N O_{i}$ accepts CRs when it adopts the cooperation strategy, i.e., in cases $(\mathrm{C}, \mathrm{N})$ and $(\mathrm{C}, \mathrm{C})$. Therefore, to achieve the optimal value of Eq. (8), $M V N O_{i}$ should accept CRs from $M V N O_{j}$ with a probability of

$$
P_{i-\text { coop }}=\frac{p_{1} \times n_{i 1}+p_{3} \times n_{i 3}}{E_{i r}} .
$$

$P_{j-\text { coop }}$ that represents $M V N O_{j}$ 's probability of accepting CRs from $M V N O_{i}$ can be calculated in a similar way.

\subsection{Discussion of Implementation Issues}

This subsection discusses several implementation related issues on the proposed fair resource sharing mechanism. As described in the previous two subsections, both MVNOs have to know the charactersitics of BRs generated by the other to calculate their NBS-based decision strategies, e.g., average number of generated BRs $\left(\lambda_{i}\right.$ and $\lambda_{j}$ shown in Table 1). In general, there are two ways to implement this pre-negotiation process in reality: (1) A centralized mode in which both MVNOs send their historical records of BRs to an authorized third-party controller, and (2) A distributed mode in which both MVNOs exchange their historical records of BRs with each other directly. Benefitted from the concept of NFV, both MVNOs and the controller may locate in the same suite of physical infrastructure. Therefore, these kinds of data and message transmission can be easily implemented by technologies like inter-process communications (IPC). Selection of different modes depends on specific requirements of application scenarios, e.g., the degree of trust between two MVNOs, and the security level of their records. We would like to skip these details in this paper, since they are beyond its scope.

After obtaining records that include the information of generated BRs, the controller (in the centralized mode) or both MVNOs (in the distributed mode) should calculate coordinates of points $D, E$, and $F$ in Fig. 6 to obtain NBSbased decision strategies. Since these coordinates represent utilities of both MVNOs in different scenarios, queueing delay of the queue in each MVNO has to be analyzed. From a queueing theory viewpoint, because the proposed system allows blocked BRs to move between queues of both MVNOs, it is a queueing system with jockeying [17]. However, except for a few simple cases that mainly correspond to the $\mathrm{M} / \mathrm{M} / \mathrm{s}$ queueing models, it is still difficult to calculate the queueing delay in general jockeying systems theoretically 


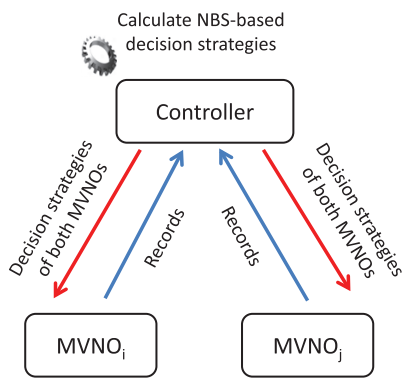

(a) Centralized mode

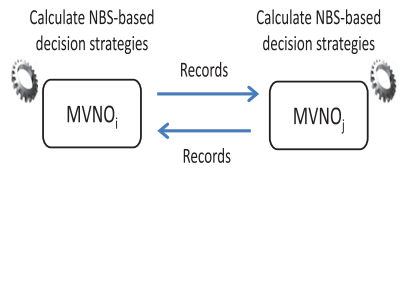

(b) Distributed mode

Fig. 7 System mechanisms of two implementation modes.

[17], [18]. Therefore, the controller or two MVNOs themselves can just simulate resource sharing processes based on exchanged records of BRs as shown in Sect. 3.3 to measure utilities of both MVNOs in reality. ${ }^{\dagger}$ Similarly, the number of received CRs listed in Table 5 can also be counted in the same simulation process by each MVNO. This kind of simulation-based implementations makes the proposed fair resource sharing mechanism not to be constrained by any specific hypothesis of existing queueing theory. Based on these metrics, the controller (centralized mode) or two MVNOs (distributed mode) calculate NBS-based decision strategies of both MVNOs. In the centralized mode, the controller broadcasts NBS-based decision strategies of two MVNOs to both of them. In the distributed mode, each MVNO understands decision strategies of itself and the other MVNO by its calculation. ${ }^{\dagger \dagger}$ With resulting NBSbased decision strategies, two MVNOs begin resource sharing process to improve their QoS. Figure 7 shows system mechanisms of the two implementation modes.

The final remaining issue is to prevent both MVNOs to deviate from the optimal NBS-based decision strategy. It is true that an MVNO does not know whether a specific $\mathrm{CR}$ will be accept by the other in advance, since the other MVNO may randomly accepts CRs with a probability. However, each MVNO come to understand the real strategy of the other by counting the percentage of its CRs accepted by the other MVNO in a period of time. Then, if any MVNO realizes that the other MVNO does not obey to the fair decision strategy, this MVNO quits from resource sharing to punish the cheating MVNO. Recall that NBS-based cooperation strategies ensure both MVNO to gain positive utilities. If there is no cooperation between two MVNOs, utilities of them decrease to zero. Since the utility of the cheating MVNO also decreases, no MVNO prefers to deviate from the optimal strategy unilaterally. Actually, this kind of punishment schemes is named "trigger-strategy" in the game theory [19].

†This is feasible because two MVNO's records of BRs have been collected by the controller or exchanged between themselves in advance.

${ }^{\dagger}$ Since two MVNOs use the same data for calculation, they get the same results.

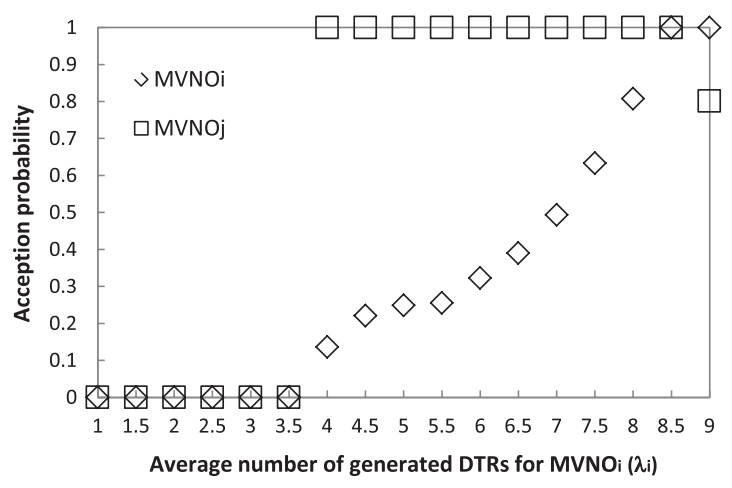

Fig. 8 Acceptance probabilities in fair resource sharing.

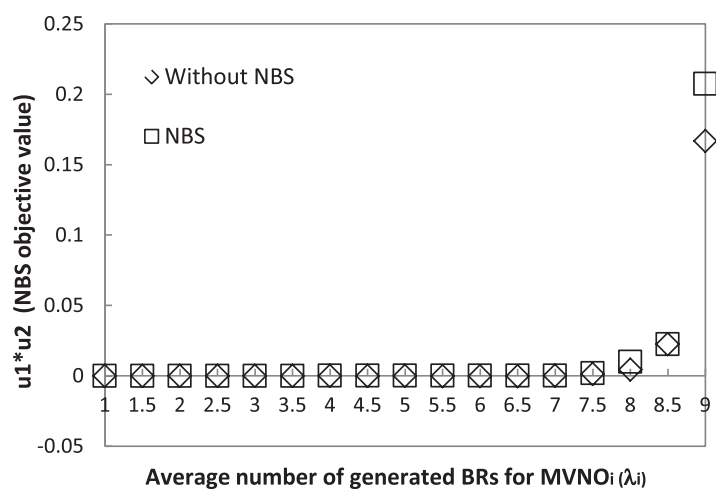

Fig. 9 Utility value of NBS.

\subsection{Numerical Results for Fair Resource Sharing}

Matlab 7.0 was used to evaluate the effectiveness of the proposed fair resource sharing mechanism. Parameter settings were the same as those listed in Table 1.

Figure 8 plots the acceptance probability of CRs for both MVNOs in the fair resource sharing. As the results show, when $\lambda_{i}<4, M V N O_{i}$ cannot benefit from the resource sharing because it was able to process its BRs immediately and never needed to send any CR to $M V N O_{j}$. Because the condition of fair resource sharing was not satisfied, both MVNOs adopted the non-cooperation strategy to reject CRs from the other. When $4<\lambda_{i}<\lambda_{j}=8.5$, it is possible for both MVNOs to benefit from resource sharing. However, $M V N O_{i}$ would receive more CRs from $M V N O_{j}$ than vice versa. Therefore, $M V N O_{i}$ tended to accept CRs with a lower probability to ensure the fairness of resource sharing. Similarly, when $\lambda_{i}>\lambda_{j}=8.5, M V N O_{j}$ tended to accept CRs with a lower probability.

Figure 9 plots the product of utilities of the NBS based decision strategy against that of the common resource sharing in which both MVNOs accept CRs from the other with a probability of one. In general, the NBS based decision strategy achieved a higher value than that of the common resource sharing (As shown in Fig. 5, the AAD of $M V N O_{i}$ is small when $\lambda_{i}$ is small. Therefore, the product of utility values of both MVNOs is quite small and is suppressed by the 
larger values in the figure.). Specifically, both MVNOs generated nearly the same number of CRs when $\lambda_{i}=\lambda_{j}=8.5$. As shown in Fig. 8, both MVNOs accepted CR with a probability of one in the NBS-based decision strategy. Therefore, utility values of both strategies are the same in this case, since there is no difference between the acceptance probability of the two strategies.

\section{Conclusion}

This paper discussed resource sharing mechanisms between MVNOs against the background of NFV. Resource sharing models were proposed to enable MVNOs to improve their QoS by cooperating with each other. An NBS-based decision strategy was also introduced to ensure the fairness of resource sharing. Finally, numerical evaluation results have validated the effectiveness of the proposed models and mechanisms.

Since this paper focused on resource sharing between two MVNOs, resource sharing models among multiple MVNOs will be investigated in the near future. Integrated resource sharing among MVNOs and their corresponding mobile devices is also an interesting topic for future study.

\section{Acknowledgment}

The authors wish to express a special acknowledgement to Dr. John Forbes Nash, Jr, the pioneer in the theory of noncooperative games. We are saddened by the death of him and his wife during the revision of this paper. This work was supported in part by the National Institute of Information and Communications Technology (NICT), Japan.

\section{References}

[1] H. Mutlu, M. Alanyali, and D. Starobinski, "Spot pricing of secondary spectrum access in wireless cellular networks," IEEE/ACM Trans. Netw., vol.17, no.6, pp.1794-1804, Dec. 2009.

[2] "Cisco visual networking index: Global mobile data traffic forecast update, 2012-2017.” http://www.cisco.com/en/US/solutions/collater al/ns341/ns525/ns537/ns705/ns827/white_paper_c11-520862.pdf, 2013.

[3] T. Frisanco, P. Tafertshofer, P. Lurin, and R. Ang, "Infrastructure sharing and shared operations for mobile network operators From a deployment and operations view," 2008 IEEE Network Operations and Management Symposium, NOMS 2008, pp.129-136, April 2008.

[4] M. Chiosi, D. Clarke, P. Willis, A. Reid, J. Feger, M. Bugenhagen, W. Khan, M. Fargano, C. Cui, H. Denf, et al., "Network functions virtualisation - Introductory white paper," SDN and OpenFlow World Congress, 2012.

[5] H.L. Cadre, M. Bouhtou, and B. Tuffin, "A pricing model for a mobile network operator sharing limited resource with a mobile virtual network operator," Network Economics for Next Generation Networks, Lecture Notes in Computer Science, vol.5539, pp.24-35, Springer Berlin Heidelberg, Berlin, Heidelberg, 2009.

[6] S. Pattanavichai, N. Jongsawat, and W. Premchaiswadi, "A pricing model and sensitivity analysis for MNO's investment decision making in 3G UMTS networks," 2010 Third International Symposium on Electronic Commerce and Security, pp.274-279, July 2010.

[7] S. Zhao, Q. Zhu, and H. Zhu, "Demand responsive pricing competition of two MVNOs in dynamic spectrum access," 2010 IEEE 12th International Conference on Communication Technology, pp.958-961, Nov. 2010.

[8] W.-C. Hong and Z. Tsai, "On the femtocell-based MVNO model: A game theoretic approach for optimal power setting," 2010 IEEE 71st Vehicular Technology Conference, pp.1-5, May 2010.

[9] I.L. Bedhiaf, O. Cherkaoui, and G. Pujolle, "Third-generation virtualized architecture for the MVNO context," annals of telecommunications - annales des télécommunications, vol.64, no.5-6, pp.339-347, 2009.

[10] O.E. Falowo and H.A. Chan, "Effect of mobile terminal heterogeneity on call blocking/dropping probability in cooperative heterogeneous cellular networks," Telecommun. Syst., vol.47, no.3-4, pp.337-349, 2011.

[11] V. Paxson and S. Floyd, "Wide area traffic: The failure of Poisson modeling," IEEE/ACM Trans. Netw., vol.3, no.3, pp.226-244, June 1995.

[12] W.E. Leland, M.S. Taqqu, W. Willinger, and D.V. Wilson, "On the self-similar nature of Ethernet traffic (extended version)," IEEE/ACM Trans. Netw., vol.2, no.1, pp.1-15, Feb. 1994.

[13] I. Norros, "On the use of fractional Brownian motion in the theory of connectionless networks," IEEE J. Sel. Areas. Commun., vol.13, no.6, pp.953-962, Aug. 1995.

[14] I. Antoniou, V.V. Ivanov, V.V. Ivanov, and P.V. Zrelov, "On the log-normal distribution of network traffic," Physica D: Nonlinear Phenomena, vol.167, no.1-2, pp.72-85, 2002.

[15] A. Goldsmith, Wireless Communications, Cambridge University Press, New York, NY, USA, 2005.

[16] D. Zhang, R. Shinkuma, and N. Mandayam, "Bandwidth exchange: An energy conserving incentive mechanism for cooperation," IEEE Trans. Wireless Commun., vol.9, no.6, pp.2055-2065, June 2010.

[17] Y. Zhao and W.K. Grassmann, "Queueing analysis of a jockeying model," Oper. Res., vol.43, no.3, pp.520-529, 1995.

[18] A. Baykasoğlu and Z.D.U. Durmuşoğlu, "Flow time analyses of a simulated flexible job shop by considering jockeying," The International Journal of Advanced Manufacturing Technology, vol.58, no.5-8, pp.693-707, 2012.

[19] Y. Shoham and K. Leyton-Brown, Multiagent Systems: Algorithmic, game-Theoretic, and Logical Foundations, Cambridge University Press, 2008.

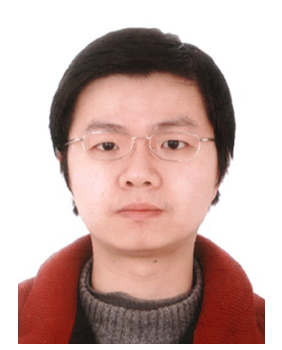

Wei Liu received the B.E. and M.E. degree in Software Engineering from Chongqing University, China, in 2006 and 2009. He is currently working toward the Ph.D. degree in Communications and Computer Engineering, Graduate School of Informatics, Kyoto University. His current research interests include overlay networks and mobile cloud computing. 


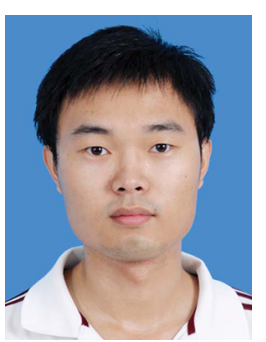

Rui Hu received the B.E. degree in Automation from University of Science and Technology of China, Hefei, China, in 2012. He received the M.E. degree in Communications and Computer Engineering, Graduate School of Informatics, Kyoto University, in 2014. His research interests was the cooperation mechanism among MVNOs.

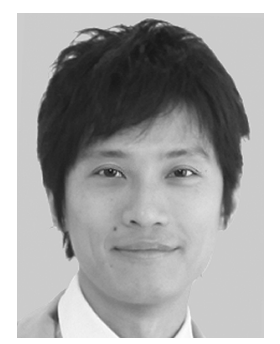

Ryoichi Shinkuma received the B.E., M.E., and Ph.D. degrees in Communications Engineering from Osaka University, Japan, in 2000, 2001, and 2003, respectively. In 2003, he joined the faculty of Communications and Computer Engineering, Graduate School of Informatics, Kyoto University, Japan, where he is currently an Associate Professor. He was a Visiting Scholar at Wireless Information Network Laboratory (WINLAB), Rutgers, the State University of New Jersey, USA, from 2008 Fall to 2009 Fall. His research interests include network design and control criteria, particularly inspired by economic and social aspects. He received the Young Researchers' Award from IEICE in 2006 and the Young Scientist Award from Ericsson Japan in 2007, respectively. He is a member of IEEE.

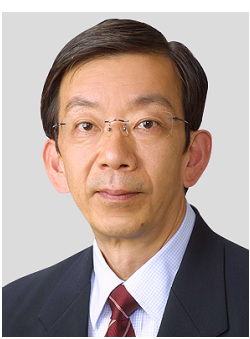

Tatsuro Takahashi received the B.E. and M.E. in Electrical Engineering from Kyoto University, Kyoto, Japan, in 1973 and 1975 respectively, and Dr. of Engineering in Information Science from Kyoto University in 1997 . He was with NTT Laboratories from 1975 to 2000, making $\mathrm{R} \& \mathrm{D}$ on high speed networks and switching systems for circuit switching, packet switching, frame relaying, and ATM. From July 2000 to Mar. 2015, he was a Professor, Communications and Computer Engineering, Graduate School of Informatics, Kyoto University. His research interests included high-speed networking, photonic networks and mobile networks. Prof. Takahashi received the Achievement Award from IEICE in 1996,the Minister of Science and Technology Award in 1998, and the Distinguished Achievement of Contributions Award from IEICE in 2011. He was a Vice President of the ATM Forum from 1996 to 1997, and the Chairman of the Network Systems (NS) Technical Group in the Communications Society of IEICE from 2001 to 2002. Prof. Takahashi is an IEEE Fellow. 